\title{
On Some DOA Algorithms for Tri-axial Geophone
}

\author{
Jan Mazur and Zbigniew Świętach
}

\begin{abstract}
In this paper a short study of some basic methods of DOA of a seismic wave using so called tri-axial geophone has been presented. The proposed methods exploit the properties of Rayleigh surface plane wave to find DOA of an incoming seismic wave using inner products of appropriately filtered signals recorded by geophones. The advantage of the proposed method is its simplicity and ease of implementation in small DSP or application processors still retaining pretty good accuracy. A number of example results for real data have been given.
\end{abstract}

Keywords - tri-axial geophone, DOA estimation.

\section{INTRODUCTION}

$\mathbf{T}$ HE direction-finding problem has been extensively studied for many years. It is a common problem formulated for different types of waves, including acoustic, electromagnetic as well as seismic waves. To find the right direction of an incoming wave i.e. direction of arrival (DOA) a great number of so called "array processing" based methods utilizes an array of appropriately spaced single sensors e.g. microphones, antennas or in case of seismic wave vertical or horizontal geophones [1],[2],[3],[4]. On the other hand specific properties of seismic waves allow for solving DOA problem using only a single device called tri-axial geophone, instead of an array of single geophones, see section II. Various methods have been developed to find DOA with the use of tri-axial geophone, though two of them seem to be the most commonly reported in the literature of the field. The first class of methods utilizes the properties of the covariance matrix (CMA - covariance matrix analysis) [5],[6],[7],[8],[9],[10], [11],[12], while the second class of methods exploits the properties of seismic waves, especially the properties of the Rayleigh surface plane wave $[13],[7],[8],[10]$. In this paper we propose a method that can be considered as an extension of those proposed in [7],[8],[14].

\section{PROBlem Formulation}

The problem is how to find (using the so called tri-axial geophone) the direction from which an unknown object to be found generates a seismic wave (see Fig. 1). The triaxial geophone is a device having 3 single geophones (usually inductors) mounted orthogonally to each other along the direction of $\mathrm{OX}, \mathrm{OY}$ and $\mathrm{OZ}$ axes of a local coordinate system, respectively. The OSVZ coordinate system is the OXYZ coordinate system rotated by angle $\theta$. Thus, the problem is to find an angle (see Fig. 1)

$$
\theta=f_{D F}(x, y, z)
$$

J. Mazur is with the Department of Electronics, Wrocław University of Technology, Wrocław, Poland (e-mail: jan.mazur@pwr.wroc.pl).

Z. Świętach is with the Department of Electronics, Wrocław University of Technology, Wrocław, Poland (e-mail: zbigniew.swietach@pwr.wroc.pl). where $x=x(t), y=y(t)$ and $z=z(t)$ are the signals measured by each of the single geophone.

In general, the movements of geophone are quite a complex mixture of incident waves of many different types and modes [15],[16],[17]. Fortunately, not all of them are of the same importance to the process of DF (Direction Finding). In this paper, according to [15],[16],[17], we have made a couple of assumptions concerning the propagation of seismic waves. Firstly, according to theory of elasticity seismic waves are described by ordinary partial differential equations with constant coefficients. Solutions for this kind of equations obey superposition principle. This approach is widely known in geophysics, where only the fundamental harmonic solutions of the above equations for a given frequency are considered. These solutions are called body compressional waves ( $\mathrm{P}$-wave) and body shear waves ( $\mathrm{S}$-wave). Usually, it can be assumed that the ground is locally homogenous and isotropic. In such a case the theory of elasticity makes it possible to simplify the solution of seismic wave propagation by introducing elastodynamic potentials. This results in new, simpler harmonic solutions of seismic wave field described by polarized waves of SH type and P-SV type. The former is shear horizontal wave and the latter is a composition of longitudinal compressional wave and transversal wave polarized in vertical plane. Secondly, in an elastic environment the waves described above interfere with each other in a sufficiently large distance from the source making two types of surface plane waves: Rayleigh wave and Love wave.

For the latter to form a thin elastic layer is required on top of ground, which in great majority of real cases is true. This means that Love wave cannot be omitted in further analysis.

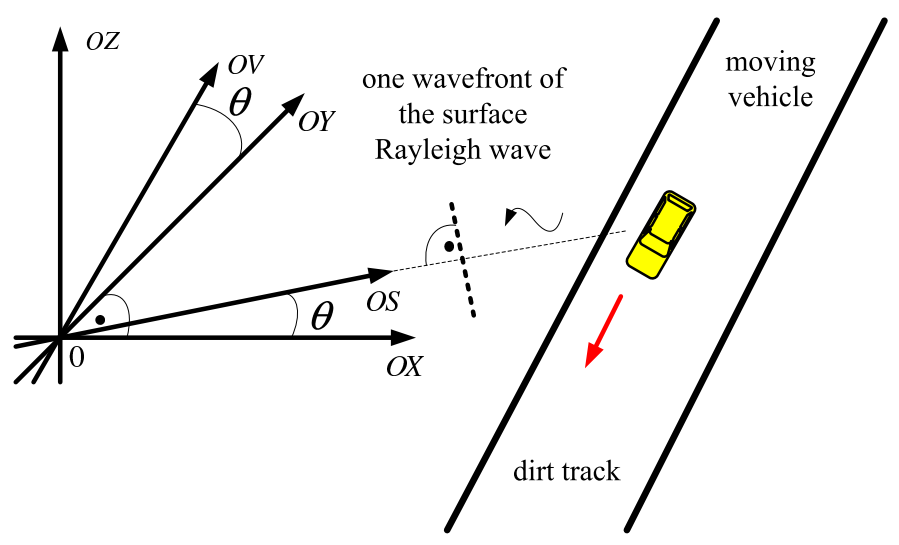

Fig. 1. The reference and rotated coordinate systems. One wave-front of Rayleigh surface plane wave that has been generated by a moving vehicle. The axes $\mathrm{x}, \mathrm{y}, \mathrm{z}$ of geophones indicate the right-handed Cartesian coordinates. The $\mathrm{z}$ axis is vertically upward oriented. 
Moreover, the considered solutions for Rayleigh and Love surface plane wave do not imply that the wave-fronts of these two waves will be parallel to each other, which was one of the assumptions in [7]. Thirdly, it is estimated [15] that in a typical ground the energy of Rayleigh wave is about $67 \%$ and Love wave is about $25 \%$ of the total energy of the seismic wave approaching the sensor. The rest of the energy comes with body waves $\mathrm{SH}$ and $\mathrm{P}-\mathrm{SV}$ randomly approaching the sensor so that to describe them a model of noise with normal (or unknown) distribution with zero mean and sufficiently small variance is applied. Also, we assume that the measurement point is positioned in a far field of the considered waves so that the model of a plane wave can be applied. Taking into account the Rayleigh model of seismic wave propagation we assumed what follows

$$
\begin{aligned}
& s(t)=s_{R}(t)+s_{N}(t)+s_{L}(t) \\
& v(t)=v_{R}(t)+v_{N}(t)+v_{L}(t) \\
& z(t)=z_{R}(t)+z_{N}(t)+z_{L}(t) \\
& v_{R}(t) \equiv 0, \quad z_{L}(t) \equiv 0
\end{aligned}
$$

where $s_{R}(t), v_{R}(t)$ and $z_{R}(t)$ are horizontal normal, horizontal tangential and vertical components of the Rayleigh surface plane wave, respectively. Similarly, lower index ŚLŠ describes the same components for Love wave projected onto the $O S V Z$ coordinate system. Index ŚNŠ describes body waves randomly approaching the detector. The left equation in (5) comes from the properties of Rayleigh wave, while the right one comes from the assumption that the vertical leakage modes of the Love wave can be neglected, because their contribution to the energy of Love wave is negligibly small. Functions $s(t), v(t)$ and $z(t)$ are displacements of the soil along $O S, O V$ and $O Z$ axes of the rotated coordinate system $O S V Z$.

The theoretical model of the Rayleigh wave states that and components are $90 \mathrm{deg}$ phase-shifted to each other and ellipticity of this wave is approximately equal to 1.5 [18],[19]. Ellipticity is the ratio of the horizontal to vertical axes of the ellipse in the elliptical motion of Rayleigh wave. However, in practice significant departures from the theoretical model are observed [18]. The components mentioned above are only roughly orthogonal, phase velocity and ellipticity are functions of frequency (dispersion appears) and consequently retrograde or prograde motion can be observed [19].

For that reason in methods of DOA estimation only the property of orthogonality of $s_{R}(t)$ and $z_{R}(t)$ components is utilized. In case of Love wave, contrary to Rayleigh wave, there is no explicit relation between its components so that no analytical relations can be given.

As a consequence if, for a given frequency, the energy of Love wave is comparable to that of Rayleigh wave, the Love wave contribution in equations 23 cannot be neglected and hence DOA methods utilizing only one tri-axial geophone are theoretically useless, because they cannot separate Love and Rayleigh wave. In [12] the authors undertook an attempt of such a separation using the array of tri-axial geophones but this method is not applicable in case of single tri-axial geophone. Using well known equations describing the rotation of the coordinate system, the equations (2),(3),(4) becomes

$$
\begin{array}{r}
s_{R}(t)+s_{L}(t)+s_{N}(t)=x(t) \cos \theta-y \sin \theta \\
v_{L}(t)+v_{N}(t)=x(t) \sin \theta+y(t) \cos \theta \\
\\
z_{R}(t)+z_{N}(t)=z(t)
\end{array}
$$

where $x(t), y(t)$ and $z(t)$ are pre-processed to be zero mean signals.

\section{CONSIDERED Methods}

Now we introduce the inner product of two real functions of the continuous variable $t$

$$
\langle x \mid y\rangle=\int_{0}^{T} x(t) y(t) d t
$$

where $T$ is the time of integration. Also, we define the inner product of two real sampled function as:

$$
\langle x \mid y\rangle=\sum_{k=0}^{K-1} x(k) y(k)
$$

where $K$ is the number of samples acquired with the sampling period $T_{S}$.

To effectively apply equations $(6,7,8)$ in the direction finding problem we further suppose the noise components have sufficiently small variances and their mean values are equal to zero. Otherwise the detected signals have to be properly smoothed and denoised. The smoothing is rather classical problem and it can be resolved using lowpass or bandpass linear filtering. The denoising, in turn, partially removes the noise located inside the pass-band of the signal considered. It can be achieved using methods described in [9],[20],[21]. Denoising method proposed in [9] is especially interesting because it was developed directly for use with seismic signals generated by footsteps.

From signal theory point of view each noise component is orthogonal to each non random signal component. It implies that inner product of any non random signal with random noise is nearly zero. Hereafter the inner products of every non random signal with any noise components are neglected.

The inner products in equations (6) and (7) with some appropriate signals are further considered. Mentioned signals are firstly properly filtered according to phase relationship between vertical and horizontal components of Rayleigh surface plane wave. The obtained results are summarised into four quite simple and effective methods of finding DOA.

From continuous time Fourier transform (CTFT) point of view the detected signals can be considered as uncountable sum of the basic harmonic oscillations. In practice, numerical approximation of the Fourier integrals consists of finite sum of isolated harmonic oscillations. Now we consider one of aforementioned basic harmonic modes with the fixed and known frequency.

Under the above conditions, inner products of the component $z(t)$ and components described in equations $(6,7)$ are equal to

$$
\left[\begin{array}{c}
\langle z \mid x\rangle \\
\langle z \mid y\rangle
\end{array}\right]=\left[\begin{array}{cc}
\cos \theta & \sin \theta \\
-\sin \theta & \cos \theta
\end{array}\right]\left[\begin{array}{c}
\left\langle z_{R} \mid s_{R}\right\rangle+\left\langle z_{R} \mid s_{L}\right\rangle \\
\left\langle z_{R} \mid v_{L}\right\rangle
\end{array}\right]
$$


The square of mutual energy of the signals (11) is equal to

$$
E_{1}^{2}=\langle z \mid x\rangle^{2}+\langle z \mid y\rangle^{2}=\left\langle z_{R} \mid s_{L}\right\rangle^{2}+\left\langle z_{R} \mid v_{L}\right\rangle^{2}
$$

since the inner product $\left\langle z_{R} \mid s_{R}\right\rangle$ is close to zero (the signals are near orthogonal). Let signals $\check{\mathrm{C}} z_{\text {shift }}(t)$ and $\check{\mathrm{C}} z_{\text {Rsift }}(t)$ be $\pm 90 \mathrm{deg}$ phase shifted version of signals $z(t)$ and $z_{R}(t)$. The sign of the phase shift depends on the motion direction of Rayleigh wave on an ellipse and retrograde as well as prograde motions are considered [18],[19]. The inner products of appropriate signals has the form

$$
\begin{aligned}
& {\left[\begin{array}{c}
\left\langle z_{\text {shift }} \mid x\right\rangle \\
\left\langle z_{\text {shift }} \mid y\right\rangle
\end{array}\right]=} \\
& {\left[\begin{array}{cc}
\cos \theta & \sin \theta \\
-\sin \theta & \cos \theta
\end{array}\right]\left[\begin{array}{c}
\left\langle z_{\text {Rshift }} \mid s_{R}\right\rangle+\left\langle z_{R s h i f t} \mid s_{L}\right\rangle \\
\left\langle z_{\text {Rshift }} \mid v_{L}\right\rangle
\end{array}\right]}
\end{aligned}
$$

The square of mutual energy of the signals (13) is equal to

$$
\begin{aligned}
& E_{2}^{2}=\left\langle z_{\text {shift }} \mid x\right\rangle^{2}+\left\langle z_{\text {shift }} \mid y\right\rangle^{2} \simeq \\
& \left\{\left\langle z_{\text {Rsift }} \mid s_{R}\right\rangle+\left\langle z_{\text {Rshift }} \mid s_{L}\right\rangle\right\}^{2}+\left\langle z_{\text {Rshift }} \mid v_{L}\right\rangle^{2}
\end{aligned}
$$

The DOA estimation is possible if the energy of Love wave for considered fixed frequency is sufficiently small in relation to the energy of Rayleigh wave, hence

$$
\left\{\begin{aligned}
E_{2}^{2} & \gg E_{1}^{2} \\
\left\langle z_{\text {Rshift }} \mid s_{R}\right\rangle^{2} & \gg\left\langle z_{\text {Rshift }} \mid s_{L}\right\rangle^{2} \\
\left\langle z_{\text {Rshift }} \mid s_{R}\right\rangle^{2} & \gg\left\langle z_{\text {Rshift }} \mid v_{L}\right\rangle^{2}
\end{aligned}\right.
$$

Only the first of the above inequalities can be explicitly verified. It is necessary to make an assumption that other conditions also holds. When DOA angle is derived, the correctness of the above assumptions is checked comparing the actual DOA angle with the previous DOA angle. The direction of the Rayleigh wave cannot change very rapidly if DOA estimations are based on adjacent time frames. When relations (15) holds the DOA estimation is derived from equations (13)

$$
-\tan \theta=\frac{\left\langle z_{\text {shift }} \mid y\right\rangle}{\left\langle z_{\text {shift }} \mid x\right\rangle}
$$

Before doing the above computations the isolated harmonic oscillations are extracted from detected signals. To do this the narrow-band filtering is performed. The detected signals are filtered by a appropriate FIR Hamming filter with the bandpass width equal to $2-3 \mathrm{~Hz}$ on the $-60 \mathrm{~dB}$ gain level. The middle frequencies of the filters are being found as the frequencies where the local maximum amplitude in the CTFT spectrum of the component $z(t)$ are observed. The local maxima are seek in the amplitude spectrum for each time frame of detected signal $z(t)$. It is important difference comparing to results obtained in [13],[5],[7],[8]. Moreover equation (16) is more general then similar equation given in [7],[8]. To explicitly derive inner product in equation (16) integration or differentiation must be performed. Using Rayleigh formulae [22] concerning inner products and fundamental properties of the CTFT transform, the above operators are replaced by simple algebraic manipulations. The mentioned equation was obtained here using only common properties of the seismic surface plane waves and basic properties of the inner products. It is in contradiction to work [7],[8] where to obtain equation like this in (16) the physical properties (accelerations and displacements) of inductive geophones are used. Our investigation is more general since formulae (16) is independent of the physical nature of detectors and may be used to signals coming from other types of seismic detectors e.g. semiconductor accelerometers.

\section{A. Method 1 - Differentiation in Time Domain}

This is our first method of direction finding using triaxial geophone. It is rather theoretical method, since numerical differentiation emphasizes high frequencies causing the processed signal more noisy. This method, however, can be practically applied when number of samples $\mathrm{K}$ is sufficiently high. Large $\mathrm{K}$ denoting the discrete time of integration in (10) is long enough, to make differentiation errors negligible. Inner product has evidently averaging property which is especially attractive when signals with additive zero mean random noise are considered. Differentiation errors just have statistically the above property. Setting

$$
z_{\text {shift }}=z^{\prime}=d z / d t
$$

we have

$$
\tan \theta=\frac{-\left\langle z^{\prime} \mid y\right\rangle}{\left\langle z^{\prime} \mid x\right\rangle}
$$

The four quadrant arctangent function should be applied to proper computation of the desired angle. Note, that the minus has to be put in the numerator of equation (18). Otherwise the obtained angle is incorrect.

\section{B. Method 2 - Integration in Time Domain}

The second method deals with integration in place of differentiation. Doing so, we eliminate the possible differentiation errors. Strictly, if $f(t)=\sin \omega t$, then $d f(t) / d t=\omega \cos \omega t$ which makes the derivative operator a kind of high-pass filter. On the contrary, the operator of integral can be considered as a kind of low-pass filter attenuating signal along $\omega$ as $\int f(t) d t=-\omega^{-1} \cos \omega t$. In the following method we take the integral of $z(t)$ instead of its derivative. It is necessary to show that the constant of integration has no influence on the result of finding DOA angle. Note, that by definition

$$
\int_{0}^{t} z(\tau) d \tau=Z(t)-Z(t)_{\mid t=0}
$$

where

$$
Z(t)=\int z(\tau) d \tau+C
$$

Taking into account (19)(19) the integration of both sides of (8) gives

$$
Z(t)+C=Z_{R}(t)+Z_{N}(t)
$$

where $C$ is the constant being a sum of all the constants of (20). From (7) and (20) we have

$$
\left\langle Z_{R} \mid v_{L}\right\rangle+C L=\left\langle Z_{R} \mid x\right\rangle \sin \theta+\left\langle Z_{R} \mid y\right\rangle \cos \theta
$$

where

$$
L=\langle 1 \mid x\rangle \sin \theta+\langle 1 \mid y\rangle \cos \theta
$$


The inner products $\langle 1 \mid x\rangle$ and $\langle 1 \mid y\rangle$ are, in fact, zeros, as $x(t)$ and $Y(t)$ are zero mean signals. Thus, (22) is satisfied for any given $C$. As a result, much like in (18)

$$
\tan \theta=\frac{\langle Z \mid y\rangle}{-\langle Z \mid x\rangle}
$$

Now, the minus has to be put in the denominator of equation (23). Otherwise the obtained angle is incorrect.

\section{Method 3 - Spectral Equivalence of Differentiation in Time}

Using classical properties of CTFT spectrum [22], we have

$$
\frac{d z(t)}{d t} \leftrightarrow j \omega \bar{Z}(\omega)
$$

where $\bar{Z}(\omega)=\mathcal{F}\{z(t)\}$ is CTFT transform of $z(t)$. The other signals recorded by geophone have analogous properties. Moreover, Rayleigh formulae for inner products [22] is applied

$$
\langle z \mid y\rangle=\left\langle\bar{Z}(\omega) \mid \bar{Y}^{*}(\omega)\right\rangle, \quad\langle z \mid x\rangle=\left\langle\bar{Z}(\omega) \mid \bar{X}^{*}(\omega)\right\rangle,
$$

where asterisks denote complex conjunction. Taking into account the above expressions, the equation (16) has the form

$$
\tan \theta=\frac{-\left\langle j \omega \bar{Z}(\omega) \mid \bar{Y}^{*}(\omega)\right\rangle}{\left\langle j \omega \bar{Z}(\omega) \mid \bar{X}^{*}(\omega)\right\rangle}
$$

\section{Method 4 - Spectral Equivalence of Integration in Time}

Using fundamental properties of CTFT spectrum [22] one can show that

$$
\int_{0}^{t} z(\tau) d \tau \leftrightarrow \bar{Z}(\omega) /(j \omega)
$$

Following the steps made in section $\mathrm{C}$ we obtain

$$
\tan \theta=\left\langle\frac{\bar{Z}(\omega)}{j \omega} \mid \bar{Y}^{*}(\omega)\right\rangle /\left(-\left\langle\frac{\bar{Z}(\omega)}{j \omega} \mid \bar{X}^{*}(\omega)\right\rangle\right)
$$

\section{RESUlts}

The real data has been collected using sensor shown in Fig. 2 using the scenario shown in Fig. 1. Each of the four methods presented in the previous chapter has been tested using real signals. Representative results presented here have been chosen from more than 30 different experiments that have been carried out with the use of moving cars as well as groups of walking and running people. In all the presented tests the sampling frequency is equal to $2 \mathrm{kHz}$. The $\mathrm{OX}$ axis of the geophone's coordinate system points out to $0 \mathrm{deg}$ while the OY axis points out to $90 \mathrm{deg}$. The vehicle is moved from left to right on a dirt track (as it was shown in Fig. 1). The tested cars were moving along the dirt road with a speed of about $35 \mathrm{~km} / \mathrm{h}$ and the CPA (Closest Point of Approach) was about $10 \mathrm{~m}$ and the distance to run for a car to each sides was about $90 \mathrm{~m}$ long (see Fig. 1). The geophone's $\mathrm{X}$ axis has been accidentally moved counter-clockwise of about 26 deg and thus it was not perpendicular to the road and hence the plots in Figs. 3-8 are not symmetrical with respect to 0

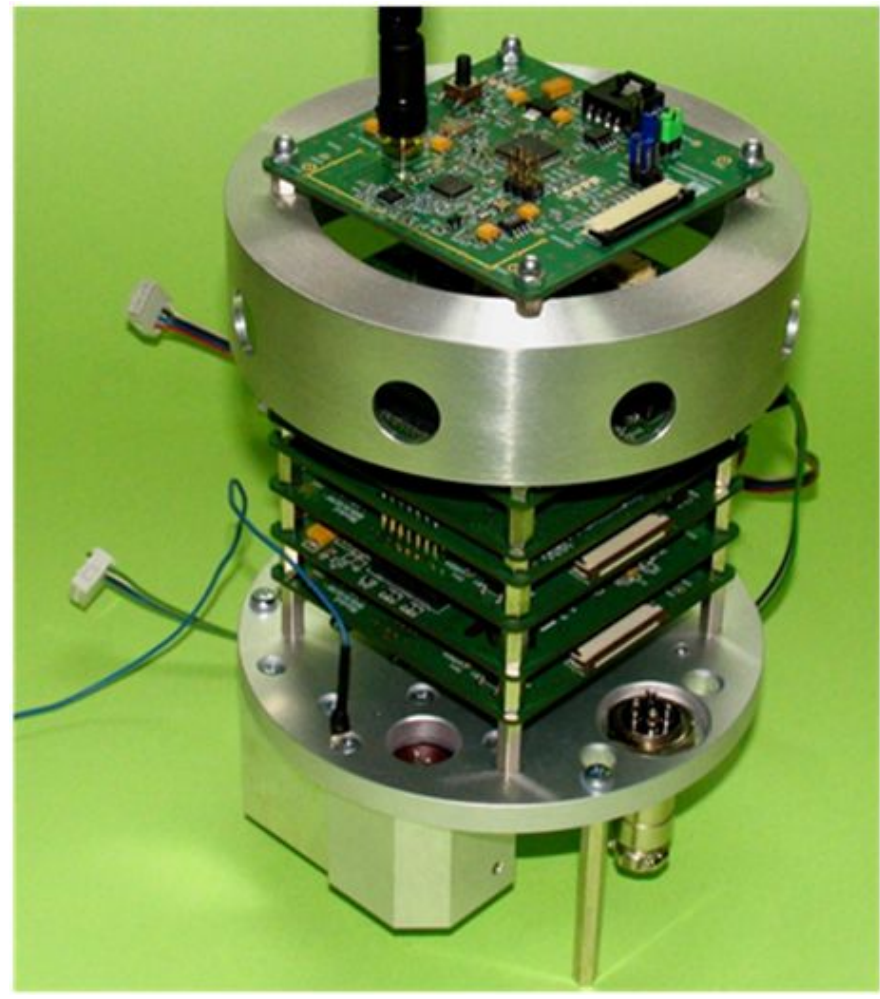

Fig. 2. The prototype of a seismic-acoustic sensor (with microphones removed). Three geophones have been mounted at the bottom of the sensor.

deg. Taking all the data concerning the measurement setup the reference plots were obtained using the following relationship

$$
\begin{array}{r}
\theta=\arctan \left(\frac{90}{10}\left(1-2 \frac{t}{t_{\max }}\right)\right)-26[\mathrm{deg}] \\
t \in\left[0, t_{\max }\right], t_{\max } \approx 18[s]
\end{array}
$$

The reference DOA (see Figs. 3-8) are plotted using the green line (no 4). The 1sec-long beginnings and endings of the signals have been removed due to unacceptably low SNR (signal/noise ratio).

Averaging vehicle position in time causes an error of about 2 deg when 512 samples are used to find the current position. (blue line in DOA plots - no 1) and about $6 \mathrm{deg}$ when 2048 samples are used (black line in DOA plots - no 2). These errors should be interpreted as minimal measurements errors. The consecutive time frames overlap roughly in 5\% of their length. The red line (no 3) in DOA plots denotes least square polynomial approximation applied to DOA estimation in case of frames of 512 samples long. The order $M$ of polynomials has been chosen with help of well known rule $\left.M \leq(1 \div 2) \sqrt{(} N_{D}\right)$, where $N_{D}$ denotes number of computed DOA angles. In presented tests this number roughly equals to $N_{D}=68$, so $M=9$ is chosen.

The least square error between the reference signal and the signal being the result of polynomial approximation procedure has been given in captions of Figs. 3-8. As expected, these errors are greater than those minimal errors mentioned above.

Sharpness of the blue line in the plots depends on the assumed frame's length. The choice of this length is a compromise between minimization of two kinds of errors mentioned 


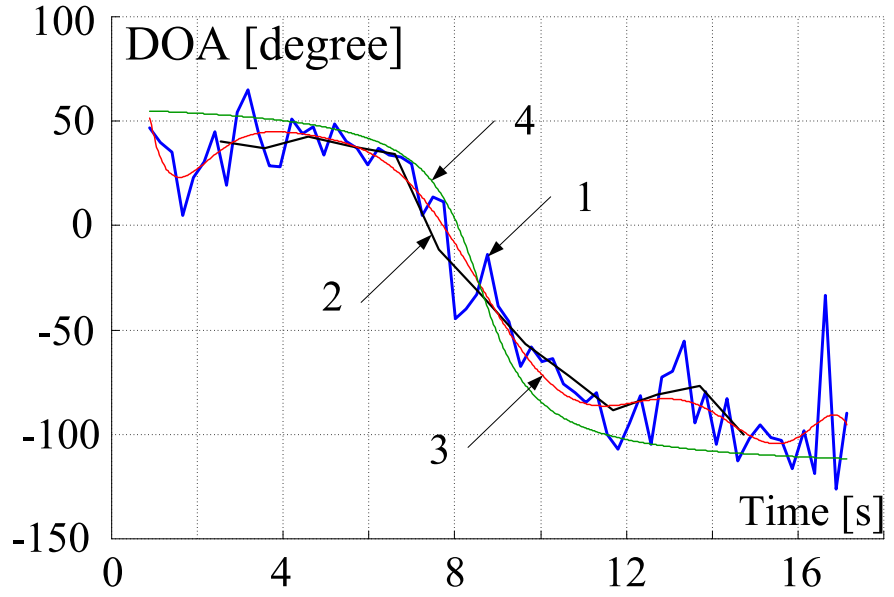

Fig. 3. DOA estimation using Method 1. A car moving from left to righ with a constant velocity equal to $36 \mathrm{~km} / \mathrm{h}$. The line no 1 (blue): 512 samples per frame, the line no 2 (black): 2048 samples per frame. The line no 3 (red): least squares approximation of the blue line. The last square error is equal to $15 \mathrm{deg}$. The line no 4 (green) is the reference signal. The last square error according to the blue line is equal to $21.5 \mathrm{deg}$.

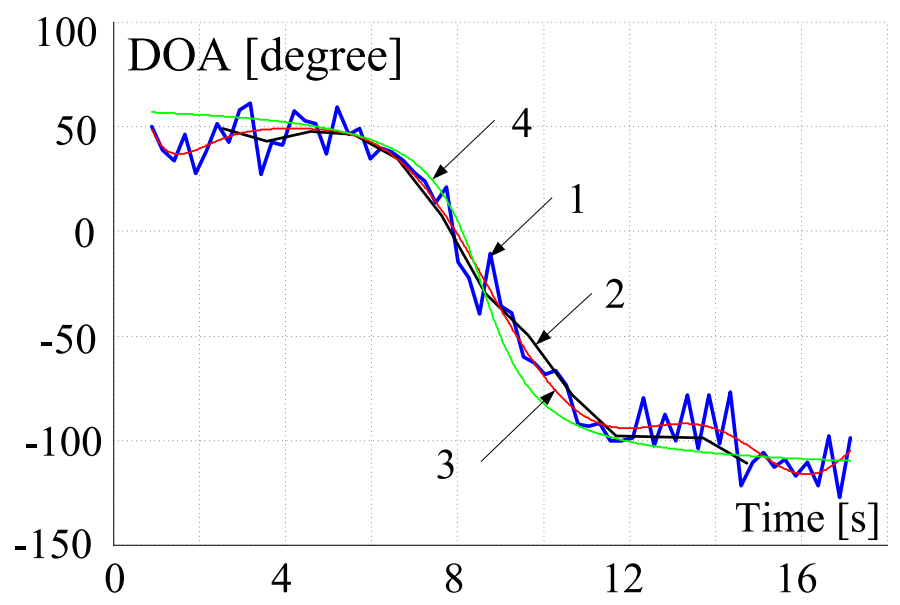

Fig. 4. DOA estimation using Method 2. The detected signals are the same signals considered in the previous example. The line no 1 (blue): 512 samples per frame, the line no 2 (black): 2048 samples per frame. The line no 3 (red): least squares approximation of the blue line. The last square error is equal to $9.6 \mathrm{deg}$. The line no 4 (green) is the reference signal. The last square error according to the blue line is equal to $13.5 \mathrm{deg}$.

above. The error of the first kind comes from averaging the car position and it grows with the length of the frame. The error of the second kind arises from the existence of zero-mean noises and, on the contrary, it declines with the length of the frame. Both kinds of errors are the result of the commonly known 'averaging' or 'smoothing' property of inner product. On one hand, it smoothes the estimated DOA curves but, on the other hand, it reduces the amplitude of unwanted noises.

The detected signals have been processed in two modes. In one of these modes signals were just stored into a flash memory of the sensor and later on they were processed using PC computer. This allowed for the usage of computationally expensive algorithms that needed powerful resources. The drawback of this is that computations are not performed in the real time. In the other mode, a simplified version of the DOA algorithm was embedded into sensor's own resources. This, in

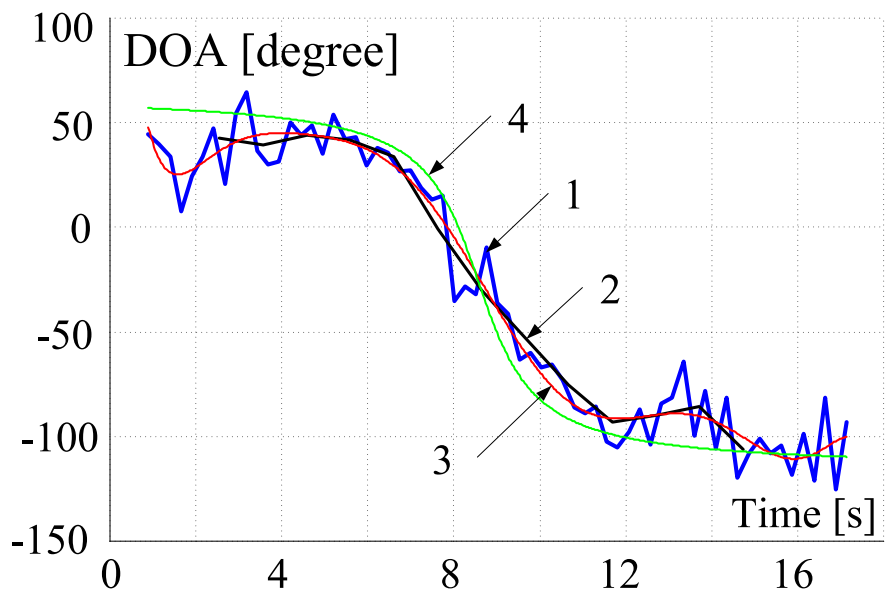

Fig. 5. DOA estimation using Method 3. The detected signals are the same signals considered in the previous example. The line no 1 (blue): 512 samples per frame, the line no 2 (black): 2048 samples per frame. The line no 3 (red) least squares approximation of the blue line. The last square error is equal to $11.5 \mathrm{deg}$. The line no 4 (green) is the reference signal. The last square error according to the blue line is equal to $17.4 \mathrm{deg}$.

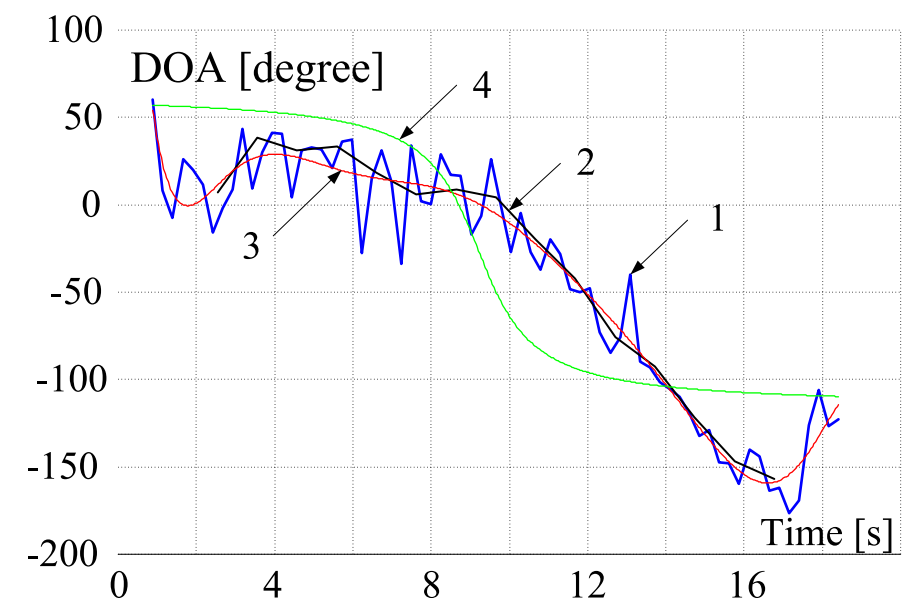

Fig. 6. DOA estimation using Method 4. The detected signals are the same signals considered in the previous example. The line no 1 (blue): 512 samples per frame, the line no 2 (black): 2048 samples per frame. The line no 3 (red): least squares approximation of the blue line. The last square error is equal to $16 \mathrm{deg}$. The line no 4 (green) is the reference signal. The last square error according to the blue line is equal to $39 \mathrm{deg}$.

turn, allowed for online computations which is very important feature in many applications. Both modes have been tested though the results given beneath concern PC computations only.

Pass-band filtering being a part of the proposed method described in the previous section is computationally rather expensive. The STM32-F105 micro-controller with some DSP capabilities that is the heart of the sensor shown in Fig. 2 is not able to make all the required computations in real time. To do this, a more powerful DSP processor would be required, most likely at the cost of much higher power consumption, which - in turn - would result in decreasing the time of operation of such a sensor equipped with the same battery.

For that reason, in the embedded (simplified) version of the proposed method the preprocessing stage, i.e. pass band filtering described in the previous section has been omitted. 


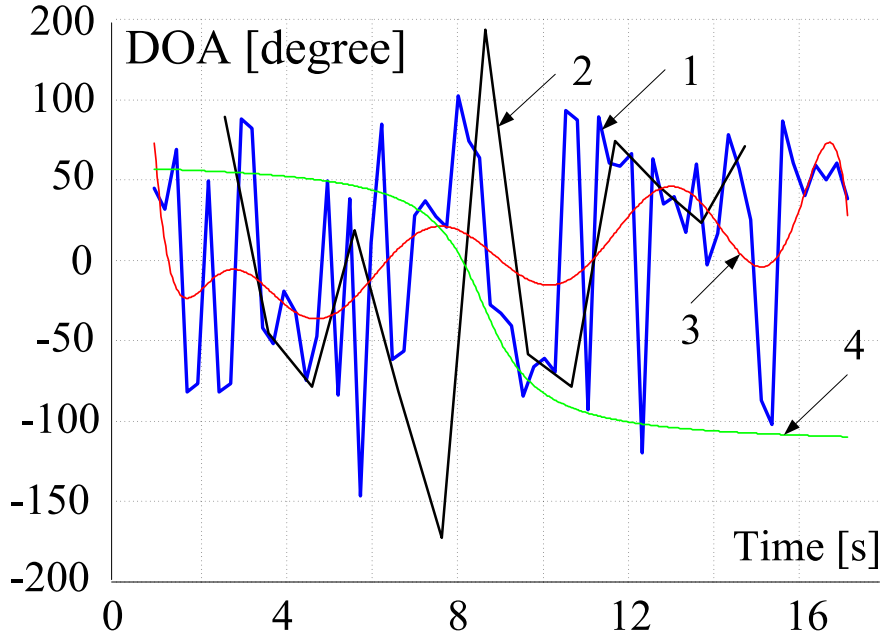

Fig. 7. DOA estimation using CMA Method. The detected signals are the same signals considered in the previous example. The line no 1 (blue): 512 samples per frame, the line no 2 (black): 2048 samples per frame. The line no 3 (red): least squares approximation of the blue line. The last square error is equal to $60 \mathrm{deg}$. The line no 4 (green) is the reference signal. The last square error according to the blue line is equal to $113 \mathrm{deg}$. The above method is obvious inadequate to DOA estimation of moving vehicles or human walking.

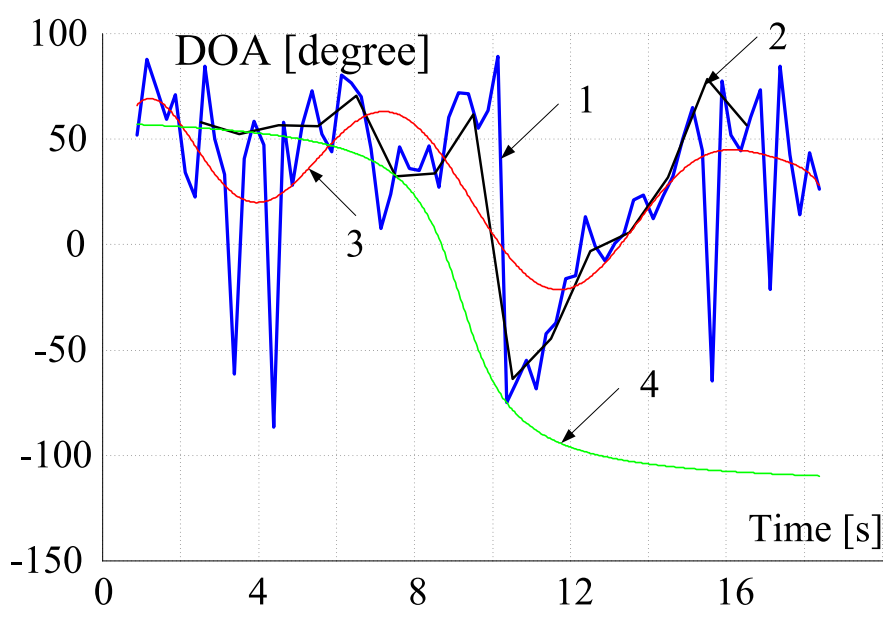

Fig. 8. DOA estimation using CMA Method with integration of the signal component $\mathrm{z}(\mathrm{t})$. The detected signals are the same signals considered in the previous example. The line no 1 (blue): 512 samples per frame, the line no 2 (black): 2048 samples per frame. The line no 3 (red): least squares approximation of the blue line. The last square error is equal to $36 \mathrm{deg}$. The line no 4 (green) is the reference signal. The last square error according to the blue line is equal to $94 \mathrm{deg}$. The above method is also inadequate to DOA estimation of moving vehicles or human walking.
The results obtained are $15 \%-25 \%$ worse than their fully processed counterparts. We believe that this level of accuracy would still be acceptable in many important applications (e.g. surveillance).

The results given in Figs. 3-8 are representative examples chosen from more than 30 different signals recorded in different scenarios. Based on the results obtained we stated that method 2 (integration in time) is the most suitable for practical usage. Note, that method 4 which is spectral version of method 2 is not as effective as method 2 . This is most likely the result of double smoothing: first (implicite)- at the stage of integration of signal (see eq. (28)) and second (explicite)- at the stage of computing the appropriate inner products.

The solutions and results presented in this paper have been compared to classical CMA method - covariance matrix analysis. To implement this method the SVD (singular value decomposition) of the appropriate signals has been performed and the desired DOA angle has been derived from eigen vectors related to the highest singular value. In fact we have implemented two different versions of CMA that differ in the way of computing the values of the covariance matrix. In the first case the input signals were used directly to find covariance matrix, while in the other case input signals were numerically integrated before they were used to find covariance matrix. In both cases the CMA turned out to be inappropriate for the class of signals we have recorded. It confirms a known suggestion [7],[8] that CMA method is adequate for high energy seismic signals (e.g. earthquakes or underground explosions). Also, CMA seems to be much more sensitive to noise when compared to methods presented in this paper.

The other methods, also partially based on the correlation analysis [13],[5],[6], seem to be worse than methods proposed in this paper although the direct comparison is impossible because of the lack of appropriate results for comparison and the lack of detailed descriptions of the methods presented in the literature.

\section{CONCLUSION}

Utilizing the properties of surface Rayleigh wave propagation to find DOA of an seismic event is the core idea for all the four methods presented in this paper. The idea, as such, is well known for years though its most common application concerned long range seismic events with high energy excitation (mainly earthquakes). In such a case surface Rayleigh wave is well formed. On the contrary, short distance, weak sources generate weak and noisy surface Rayleigh wave with many random waves approaching the detector.

To improve SNR (signal to noise ratio) of recorded signals we have proposed a preprocessing block of narrow-pass-band filters with their base frequencies chosen based on some spectral properties of vertical component, i.e. $z(t)$. Also, assuming the model of seismic wave described by equations (6), (7) and (8) we derived inequalities (15). Further, unlike in [7] and [8] we derived 16 with no assumption on physical properties of geophones used.

Unfortunately, at this stage of development the proposed method of signal preprocessing can only be implemented on PC platforms or on platforms equipped with powerful DSP processors. On the other hand, a simplified version of method 2 can be successfully implemented even on small DSP processors if only the proposed filtering procedure would be omitted. This results in increasing the error rate to about $20 \%$ but the implementation is very easy and the code can be vary fast. For example, the code running $72 \mathrm{MHz}$ STM F105-family processor allows for finding 8 DOA angles/sec using frames of 512 samples long. 


\section{ACKNOWLEDGEMENT}

The authors want to acknowledge Sławomir Sambor, Zbigniew Sałamacha and Paweł Bardowski for designing and making the hardware of the sensor. Also we wish to thank all the people helping us with real data collection.

\section{REFERENCES}

[1] J. Chen, Y. Kung, and R. Hudson, "Source localization and beamforming," IEEE Transactions on Signal Processing, vol. 19, no. 2, pp. 30-39, March 2002.

[2] P. Stoica and A. Greshman, "Maximum likelihood doa estimation by data supported grid search," IEEE Signal Processing Letters, vol. 6, no. $10,1999$.

[3] P. Stoica and K. Sharman, "Maximum likelihood methods for directionof-arrival estimation," IEEE Transactions on Acoustic, Speech and Signal Processing, vol. 38, no. 7, 1990.

[4] R. Roy and T. Kailath, "Esprit-estimation of signal parameters via rotational invariance techniques," IEEE Transactions on Acoustic, Speech and Signal Processing, vol. 37, no. 7, 1989.

[5] J. Bobbitt, "Three component rayleigh wave filter," America Oil and Gas Journal, pp. 72-77, 1986

[6] E. Smith et al., "Measurement and localization of the interface wave reflections from a buried target," Journal Acoustical Society of America, pp. 2333-2343, 1998.

[7] J. Stafsudd, S. Asgari, M. Ali, C. Chen, R. Hudson, F. Lorenzelli, K. Yao, and E. Taciroglu, "Analysis, implementation and application of acoustic and seismic arrays," Acta Automatica Sinica, vol. 32, no. 6, 2006.

[8] J. Stafsudd, S. Asgari, R. Hudson, K. Yao, and E. Taciroglu, "Localization of short-range acoustic and seismic wideband sources: Algorithms and experiments," Journal of Sound and Vibration, vol. 312, pp. 74-93, 2008.

[9] V. Reddy, D. Venkatraman, A. Khong, and B. Ng, "Footstep detection and denoising using a single tri-axial geophone," in Conference on Circuits and Systems, Asia, December 2010, pp. 1171-1174.
[10] D. Venkatraman, V. Reddy, and A. Khong, "A study of the ambiguity problem in footstep bearing estimation using tri-axial geophone," in 8 th International Conference of Information, Communication and Signal Processing (ICICS), December 2011, pp. 1-5.

[11] D. Venkatraman, V. Reddy, A. Khong, and B. Ng, "Polarization-cumenergy metric for footstep detection using vector-sensor," in International Conference on Technologies for Homeland Security (HST), November 2011, pp. 196-201.

[12] R. Kirlin, J. Nabelek, and G. Lin, "Triaxial array separation of rayleigh and love waves," in IEEE Proceedings of ASILOMAR-29, 1996, pp. $722-725$.

[13] S. Stotts, R. Gramann, and M. Bennett, "Source bearing determination from a tri-axial seismometer using rayleigh wave propagation," Journal of Acoustical Society of America, pp. 2003-2012, May 2004.

[14] Z. W. J. Mazur, "Efficient doa estimation using tri-axial geophon," in The International Conference on Signals and Electronic Systems ICSES' 2012, Wrocław, 2012.

[15] Z. Kasina, Seismic Reserach Methodology. Kraków: GSMiE PAN Publishing, 1998, in polish.

[16] _ - Seismic Signal Theory. Krakow: AGH Publishing, 2009, in polish.

[17] O. Novotny, Seismic Surface Waves. Bahia, Salvador: Instituto de Geociencias, 1999

[18] M. Hobiger, N. Bihan, C. Cornou, and P. Bard, "Rayleigh wave ellipticity estimation from ambient seismic noise using single and multiple vector-sensor techniques," in 17th European Signal Processing Conference, Nagoya, Japan, August 2009, pp. 2037-2041.

[19] _ _ "Multicomponent signal processing for Rayleigh wave ellipticity estimation," IEEE Signal Processing Magazine, pp. 29-39, April 2012.

[20] D. Donoho, "De-noising by soft tresholding," IEEE Transactions on Information Theory, vol. 41, no. 3, pp. 613-627, May 1995.

[21] C. Taswell, "The what, how, and why of wavelet shrinking denoising," Stanford University, Tech. Rep. CT-1998-09, September 1998.

[22] J. Szabatin, Fundamentals of Theory of Signals. Warszawa: WKiE, 1982, in polish. 